\title{
Mosaics of polynomial transformations giving a patient specific registration to reduce breathing motion artefacts
}

\author{
John Jones, Member, IEEE, Emma Lewis, Ashrani Abd Rahni, Member, IEEE, Veni Ezhil, and Kevin Wells
}

\begin{abstract}
Nuclear Medicine (NM) imaging is an important diagnostic tool, used widely in the field of oncology amongst others. However, NM images suffer from significant blurring due to inevitable patient motion, such as breathing, coughing and other voluntary or involuntary movement. Advances in detector technology and reconstruction techniques have led to a steady improvement in NM spatial resolution and the problems posed by patient motion are therefore becoming increasingly significant, particularly for PET/CT. Many correction schemes will require gated images to be aligned. This paper describes a method of registering major organs in piecewise fashion called virtual dissection. Results from processing synthetic data and one set of patient data are presented. A key feature is the possibility to use a mixture of registration techniques on a single set of data and combine the results into a single set of output images.
\end{abstract}

Index Terms_registration, breathing, blurring, ICP, SPECT, PET, NCAT, XCAT, AIR

\section{INTRODUCTION}

Nuclear Medicine (NM) imaging is an important diagnostic tool, used widely in the field of oncology amongst others. However, NM images suffer from significant blurring due to inevitable patient motion, such as breathing, coughing and other voluntary or involuntary movement during the long ( $\approx 10-30$ minutes) acquisition times. Advances in detector technology and reconstruction techniques have led to a steady improvement in NM spatial resolution and the problems posed by patient motion are therefore becoming increasingly significant, particularly for PET/CT.

If a sequence of CT images were available, then an abdominal thoracic registration method could be used to build an internal breathing model with deformation fields that could be applied to corresponding gated NM image data. One such approach is to use a particle filter to adapt the registration based breathing model to previously unseen internal organ motion[1]

This paper describes a method of registering major organs in piecewise fashion called virtual dissection. Two registration methods have been compared in the context of virtual dissection: Iterated Closest Points (ICP)[2]. and Automated Image Registration (AIR)[3]. Results from processing synthetic data and one set of patient data are presented. A

J. Jones, E. Lewis, A. Abd Rahni and K. Wells are at the Centre for Vision, Speech and Signal Processing, University of Surrey, Guildford, Surrey GU2 7XH, UK. e-mail: j.jones e.lewis a.abdrahni k.wells @surrey.ac.uk

M. Ezhil is at St Luke's Cancer Center, The Royal Surrey County Hospital NHS Trust, Guilford GU2 7XX, UK. e-mail: veniezhil@nhs.net key feature is the possibility to use a mixture of registration techniques on a single set of data and combine the results into a single set of output images.

Applying a single affine transformation to an entire torso does not lead to a solution sufficiently accurate for breathing motion correction [4]. This work has been undertaken to consider how to transform each organ independently, as part of a piecewise or organ by organ approach such as virtual dissection.

\section{METHOD}

An implementation of ICP, was developed specifically for the virtual dissection procedure described here. $\operatorname{ICP}(6)$ was developed in Matlab, based on a $\mathrm{C}_{+}+$implementation by Bergstrom and Shechter [5]; the parenthesis indicating rigid transformations with 6 degrees of freedom (dof). ICP(12) uses the result of $\operatorname{ICP}(6)$ as a starting value and provides affine solutions (12 dof). It is a Matlab implementation based on Du et al.[7]. For synthetic data and the majority of the organs in the patient data, ICP(12) was used but, for patient organs partly outside the field of view, ICP(6) was used. This was because ICP(12) scaled incomplete organs, which were significantly different in size between the extremes of the breathing cycle. A second technique, Automated Image Registration (AIR 5.2.6), was used to register binary images of individual organs. These were run separately for each breathing phase and each organ.

For both registration techniques (ICP and AIR) the first phase of the breathing cycle was registered, as the reference image, against each of the other phases in the cycle. Multiple registrations of individual organs were carried out. The displacement fields resulting from those CT registrations could then be applied to the NM images having the appropriate phase in the cycle and the results summed to form an NM image with improved signal to noise ratio and reduced blurring. However in this paper we confine the analysis to the CT data.

The method for registration by virtual dissection is described in summary by Figure 1 .

It is assumed, without loss of generality, that deformation occurs during a breathing cycle of $n$ phases starting at fullinspiration. The first phase is paired with each of the other $(n-1)$ phases. Figure 1 shows the processing of one such pair of images. The numbered action boxes are described in more detail below. 


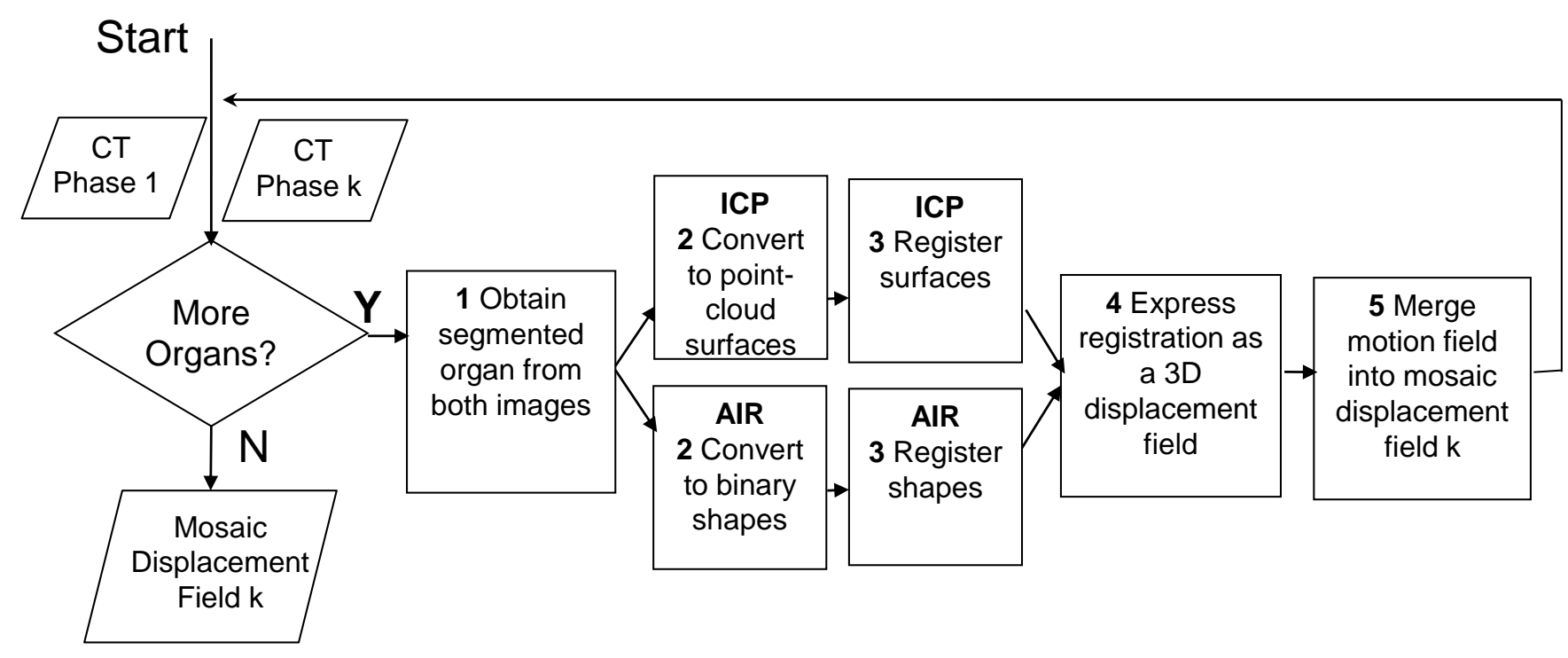

Fig. 1. Process flowchart covering the ICP registrations.

1. Obtain Segmented Organ. The method assumes the availability of a fully segmented set of images. Currently these include individual segmented images of heart, liver, gall-bladder, spleen, kidneys, stomach and contents, intestines, lungs, spine, shoulder bones, sternum, skin and ribs.

2. Convert to point-cloud representation. For ICP, the segmented organs for each pair of images are converted to surfaces using morphological operations such as erosion and subtraction. The surfaces are converted to point-cloud representation. For AIR, the process is similar but stops short of converting to surfaces and presents the entire shape as a binary image.

3. Register Surfaces. The MATLAB implementation of ICP with twelve degrees of freedom $(\operatorname{ICP}(12))$, developed for this work, is based on point-cloud representations of the shapes being registered. 3D rigid and affine spatial transformations are usually expressed as a $3 \times 3$ matrix and a 3 component translation. This is a convenient shorthand for a set of 3 first-order, or linear, equations in three variables. ICP expresses its results in this manner. The Automated Image Registration tool (AIR) develops this idea by extending the set of linear equations, equivalent to an affine transformation, to polynomials of higher order in the same three variables. The shorthand of the $3 \times 3$ matrix is extended to a table of polynomial coefficients, held in a canonical order, which is used to provide a spatial transformation more flexible than affine. For AIR, the results of the registrations are analogous to the ICP matrix but is $3 \times n$ where $n$ depends upon the order of the polynomial model in use. One advantage of this approach is that even with 4th or 5th order solutions, having 105 and 168 parameters respectively, the numbers of parameters involved is considerably smaller than the number of parameters associated with, for example, cubic spline based registrations. An assumption of the ICP surface registration is that each organ is registered, taking into account only the surface, and the resulting transforma- tion is extended into the interior of the organ.

4. Express registration as a displacement field. In the case of ICP the affine matrix plus translation vector are a concise description of each organ's transformation; in order that several such transformations can be combined, the representation is changed into a displacement field by evaluating the transformation on each voxel center and retaining only those voxels which lie within the relevant organ on the reference image. For AIR, the polynomial coefficients are available as part of the output. These coefficients are used to evaluate the transformation on voxel centers and a selection retained as for ICP.

5. Merge registration into mosaic displacement field. A mosaic displacement field is maintained for each of the nine pairs of images. With the synthetic data, the boundaries of the organs are soft in that sometimes a particular voxel will be indicated as part of more than one organ. We have catered for this possibility as follows. Corresponding to each organ or component included in the registration is a region of the mosaic displacement field. The range of each of these regions is defined by the shape of the organ or component in the reference phase. Thus overlaps between component regions of the mosaic are minimal and confined to the edges of components. A simple scheme is currently adopted whereby the mosaic displacement field consists of a three dimensional sum of translations together with a normalizing component. Thus overlaps result in an averaging of component displacements.

\section{A. Source data}

1) Simulated Data: XCAT [8] is an anthropomorphic, breathing computational phantom. The primary output is a set of linear attenuation maps and emission maps. The attenuation maps are converted to Hounsfield units shifted by 1000 so that the image background has value zero. XCAT's Vector Displacement Maps (VDMs) give, for each 
voxel of tissue in the reference frame, the coordinates of the same tissue in subsequent frames. Thus it is possible to trace the ground truth motion of any voxel throughout the cycle of images. The emission maps are not used in the work presented here.

2) Patient Data: One set of patient data has been processed. The data are from dynamic CT captured from a patient to whom a contrast agent had been administered.( $92 \mathrm{mls}$ of Niopam 300 at a flow rate of $2 \mathrm{mls} / \mathrm{sec}$ starting approximately 10 seconds before the start of $4 \mathrm{D}$ scan) The data form a single, ten phase cycle.

\section{B. Validation}

Of the two validation methods the Consistently Labelled Fraction (CLF) is calculated on resliced images with a given voxel size whilst the VDM based measures deal in fractional millimetres. The CLF is calculated for both patient and XCAT data while the VDM based measure is only available for XCAT data.

1) Consistently Labelled Fraction measure: This measure, sometimes known as the Jaccard statistic, is used to evaluate the results of the serial individual organ registrations.

Considering the reference image in its coordinate system and the source image registered into the same coordinate system, the two images approximately overlap and the overlap (intersection) is referred to as the consistently labelled part. If the image components are $A$ and $B$ and \#() is an operator returning the number of voxels of the operand, then the measure selected here is the fraction of voxels consistently labelled or $\mathrm{C}$ where

$$
C=\frac{\#(A \cap B)}{\#(A \cup B)}
$$

Since the statistic is determined from the registered images themselves it serves as an end-to-end validation which is available with both XCAT simulated data and patient data.

2) Analyses using XCAT Vector Displacement Map output: Five sets of coordinates were chosen to be treated as lesion centres in the reference frame. These were 'placed' so as to be near organ boundaries representing the positions considered to cause most difficulty in clinical diagnosis. These logical starting positions in combination with the XCAT phantom VDMs yielded the positions of the lesion centers in each of the other nine frames. The motion fields resulting from the registrations were used to determine where the lesions should have been in frame 1 if the registrations were perfect. The measure used to evaluate the registration results was the diameter of the set of such centers of the lesions. The "diameter" of the set of lesion centers is the diameter of the smallest sphere which can contain all the centers of the lesions. (This is not related to the diameter of the lesions themselves.) If the registration processes were perfect, the post registration diameter of the centers would be zero since the centers would be coincident. That set of values is contrasted with the diameter of the sets of centers without registration.
In addition, the average absolute difference between the displacement provided by the VDM ground truth and the relative displacement resulting from the corresponding registrations was measured.

\section{Results}

\section{A. Registration quality}

1) Evaluation of registration quality using CLF: The registration quality of AIR and ICP compared to no registration is shown in Figure 2 for XCAT data and Figure 3 for patient data. The figures show how the CLF varies from frame to frame for several organs. The patient data was semiautomatically segmented and some components were not discernable. However the major organs are present for both XCAT and patient data. For the smaller components, notably the gall-bladder and sternum, the 'before registration' (red line), drops close to zero for the phases that represent the largest motions; when the unregistered organ is superimposed on the reference organ coordinate system at maximum displacement (e.g. phase 1 with phase 6) there is a small intersection and a relatively large sized union. For larger organs which translate no further than some small organs the effect is not so pronounced. In the patient data (Figure 3 ) some organs were only partly visible in the CT images and the portion visible varied throughout the breathing cycle (liver, spleen and kidneys). In the ICP(12) method the different sized visible components were rescaled to match sizes and when resliced the organs were of incorrect size. The ICP results were improved by using $I C P(6)$ to obtain a rigid solution instead of $I C P(12)$ for those partly visible organs. It is fortunate that the partly visible organs were among those which vary little in volume. It would be a problem if the lungs were only partly visible.

Where there is little or no movement of an organ, such as shoulders or spine, the three lines coincide and the CLF is always very close to one. Where one might expect a degree of non-linear motion such as with lungs, intestines and ribs AIR results in a CLF value closer to unity. Otherwise AIR and ICP CLF results show a reassuring degree of agreement, with AIR showing a slight improvement over ICP.

2) Final spread of the lesion centres: Table I shows the pre and post registration spread of the sets of lesion centres. A perfect set of registrations would return the lesion centres to the frame 1 coordinates of the starting position, the final diameter would be zero as would the percentage separation remaining. The final diameters and percentage separation remaining show ICP gave better results than AIR.

3) Average absolute distance between homologous points: Figure 4 shows, for each organ registered except the skin, the average absolute distance between all pairs of homologous points. This measure is available only with the XCAT simulation data. The average absolute differences are, from the overall average section of the figure, below $3.25 \mathrm{~mm}$ - the voxel size of the simulated data. The relatively poor results for skin have not been included. They are thought to be unrepresentative because, as a single organ, it includes the largest dimensions and volume yet has 

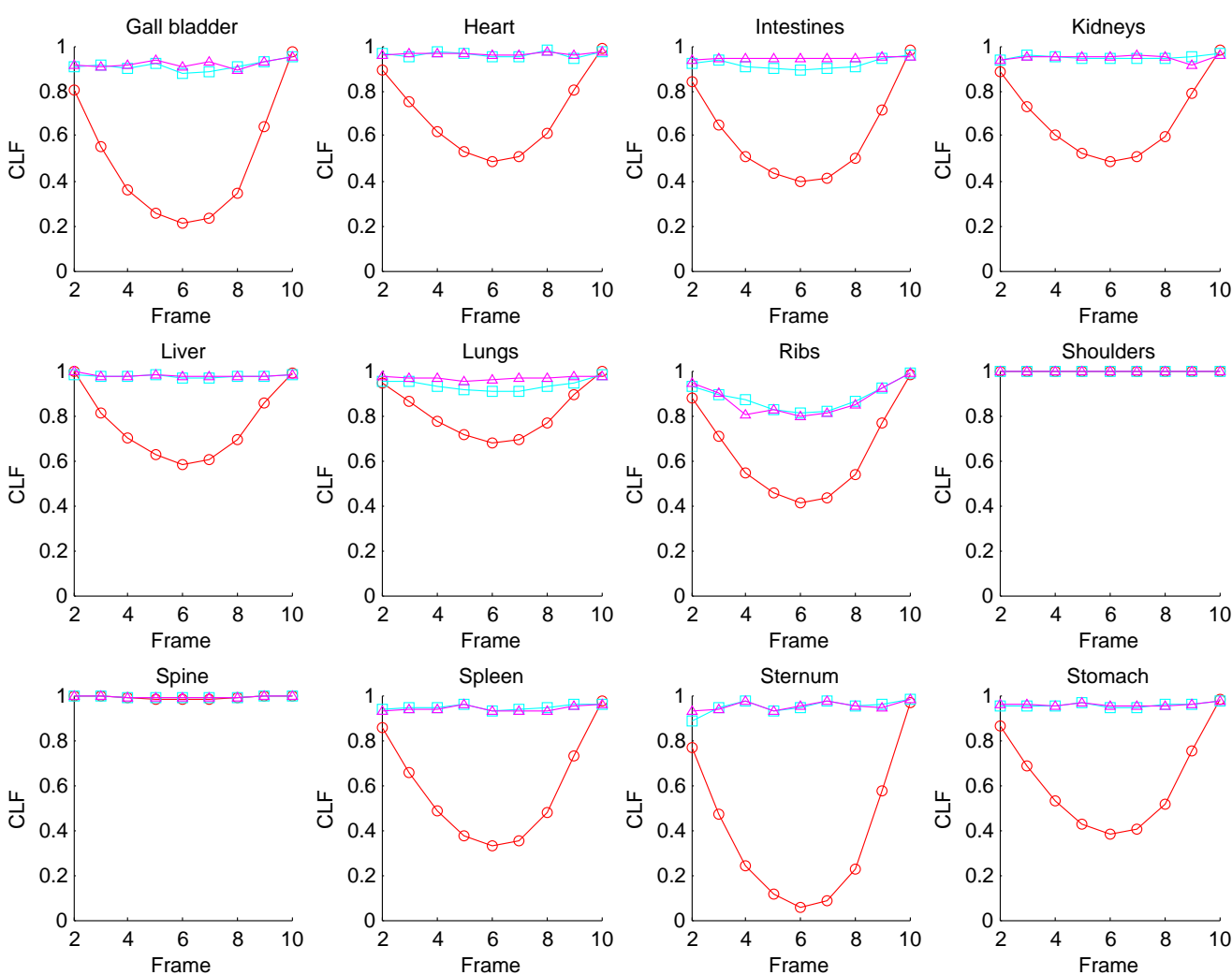

Fig. 2. XCAT data from a single breathing cycle of 10 frames. Individual organs pre and post registration consistently labelled fraction statistic. Red: No registration, Cyan: ICP, Magenta: AIR. The reference or model image is frame 1. ICP(12) used except for kidneys, spleen and liver where ICP(12) was used.
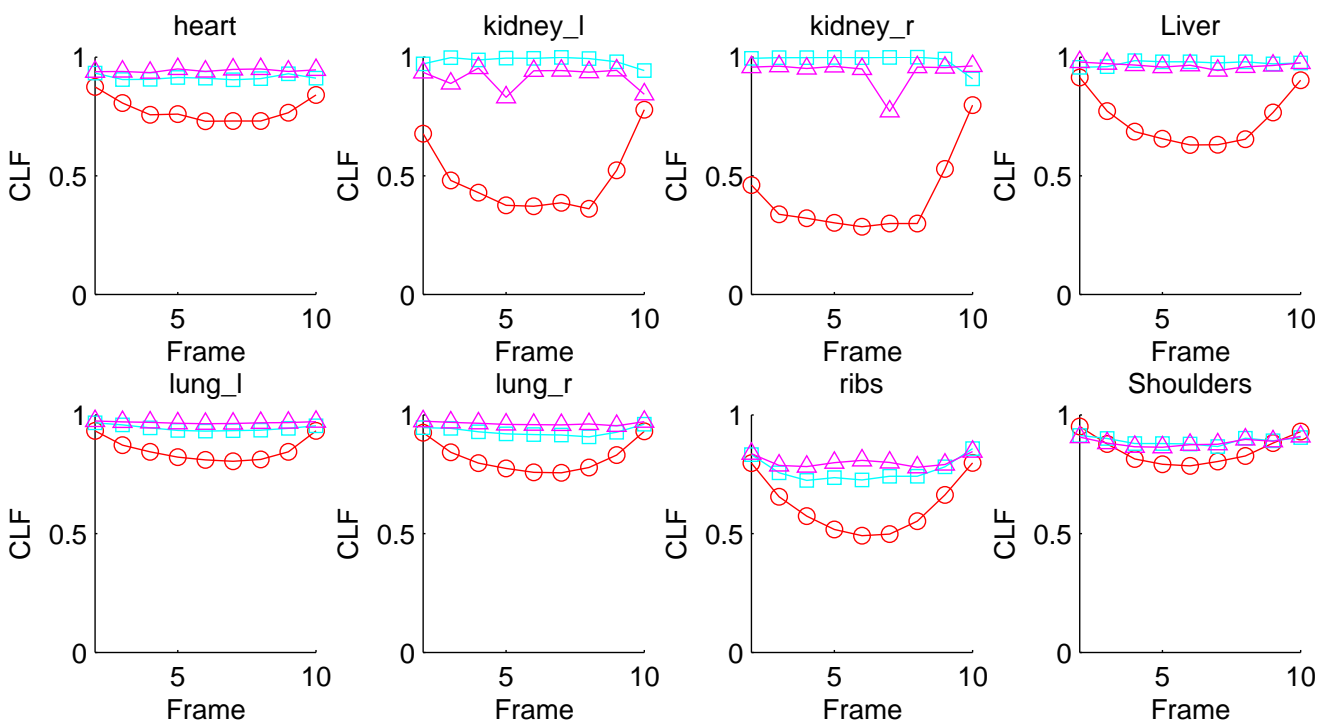

lung_r

ribs
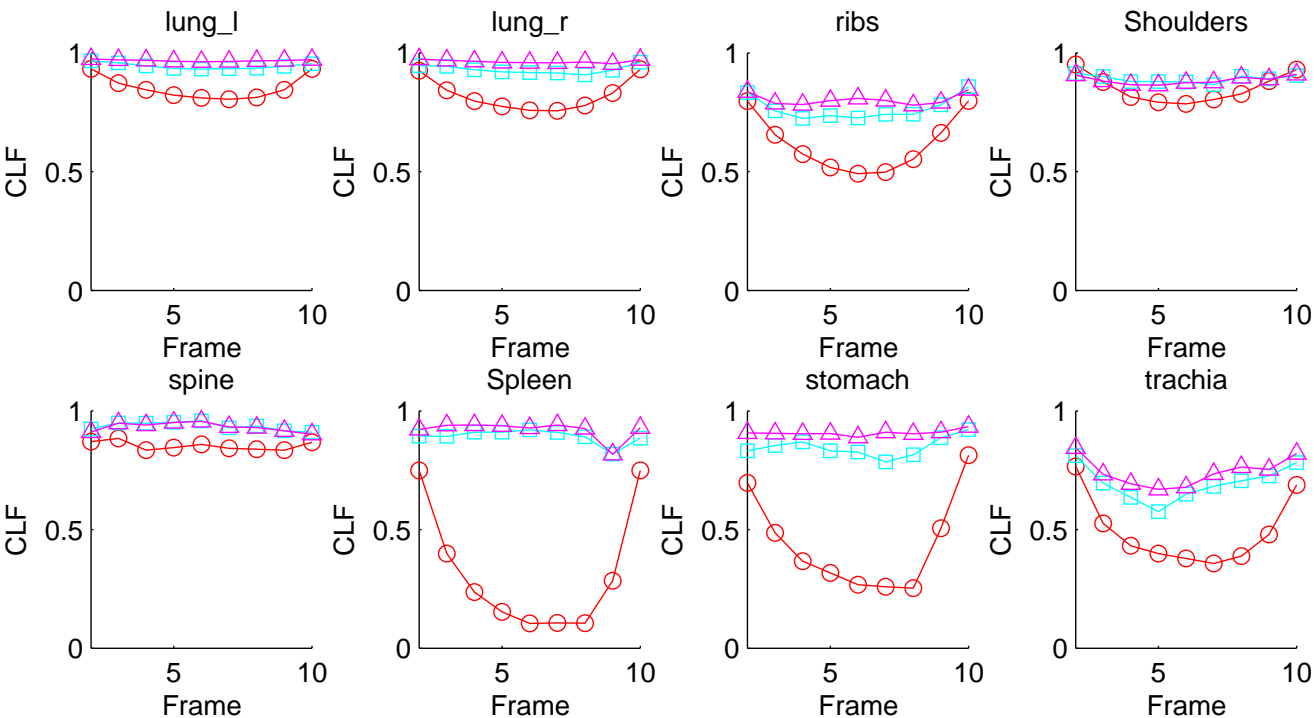

Fig. 3. Patient data from a single breathing cycle of 4D CT comprised of 10 frames. Individual organs pre and post registration consistently labelled fraction statistic. Red: No registration, Cyan: ICP, Magenta: AIR. The reference or model image is frame 1. 
TABLE I

Pre and Post REGISTRATION SPREAD OF THE LESION CENTRES

*PREREGISTRATION DIAMETER OF LESION CENTRES(VOXELS),

**POSTREGISTRATION DIAMETER OF LESION CENTRES(VOXELS),

${ }^{* * *}$ FINAL DIAMETER AS PERCENTAGE OF INITIAL DIAMETER.

\begin{tabular}{|c|c|c|c|c|c|c|}
\hline & & & ICP A & ine(12 dof) & & AIR \\
\hline & & & & & & $\%$ \\
\hline Lesic & esion & Initial & Final & Separation & Final & Separation \\
\hline Numk & rPosition & Diameter* & Diameter & Remaining*** & Diameter & Remaining ${ }^{* * \star}$ \\
\hline 1 & Center of top & 6.76 & 0.63 & 9.33 & 2.83 & 41.90 \\
\hline 2 & Base of R.Lung. Center & 5.3 & 1.31 & 24.76 & 1.3 & 25.62 \\
\hline 3 & Liver near boundary of R.Lung and Ribs & 6.76 & 0.63 & 9.31 & 2.90 & 42.82 \\
\hline 4 & Center of L.Lung above Heart level & 4.99 & 0.98 & 19.73 & 3.10 & 62.19 \\
\hline 5 & Top-center of R.Lung near Chest wall & 2.73 & 0.56 & 20.48 & 1.02 & 37.19 \\
\hline
\end{tabular}

a single voxel thickness. Small variations in the rotation component give relatively large differences in displacement in the regions furthest from the origin.

One problem with the CLF measure is that all voxels in the intersection count equally in calculating the statistic whilst some homologous points might be a considerable distances apart. The average absolute distance figures indicate that this is not the case for our XCAT simulation data. The magnitude of breathing motion encountered in the simulated data is not large and is larger than found in the patient data. Translations are usually no more than $20 \mathrm{~mm}$ and rotations are all less than 5 degrees and usually much smaller, therefore it seems reasonable that improvements in CLF are reasonable indicators of improved registration for this situation. AIR offers some degree of control over the order of the polynomial used to express the transformation. The result of reducing one of the precision parameters of AIR will be that higher orders of polynomial will be employed, if necessary, to meet the increased precision indicated by the reduced parameter. For organs which exhibit non-linear deformation, higher order polynomials ( more parameters) lead to a better registration (CLF value closer to 1). The agreement between the CLF statistics from AIR(affine) and ICP(affine) for the individual dissected organs demonstrates that ICP is equivalent to AIR(Affine), at least for the datasets used here. In the case of the lungs, shown in Figure 5, where motion cannot be described exactly by a linear solution, the higher order polynomials of AIR offer an improvement over the affine solutions of both AIR5 and ICP. In the case of the simulated lungs this leads to a $2 \%$ increase in the number of voxels in the intersection. (Going beyond the 5th order produced no further improvement.)

An objective assessment of the quality of the final images re-assembled in the virtual dissection process is more difficult for patient data in the absence of ground truth as reference. A subjective measure of the registration quality is a ten frame movie made with frame 1 being coronal, sagittal and transverse views centered on a selected voxel of the reference image. The no registration view is placed alongside the post registration view. The subsequent 9 frames are similar views centered on the same voxel but from successive un-registered and post registration views. Perfect registration would result in ten identical post registration views. The residual movement that is seen is a subjective measure of the registration quality. The movies show a significant improvement over the no registation situation for both AIR and ICP [6].

\section{CONCLUSIONS AND FURTHER WORK}

The virtual dissection approach considers breathing motion on an organ by organ basis. It has been demonstrated using both ICP and AIR separately. It is possible to choose the registration technique at an individual organ level and combine them in a single resliced file; though no such results are presented in this paper.

In the case of simulated data of the lungs and other regions, where motion cannot be described exactly by a linear solution, the higher order polynomials of AIR offer a slight improvement over the affine solutions of both AIR and ICP. In the case of the lungs this leads to a $2 \%$ increase in the number of voxels in the intersection.

An objective method for simulated data is possible because XCAT2 provides voxel by voxel motion between the reference image and the frames paired with it. Figure 4 shows that for $3.25 \mathrm{~mm}$ voxels sub voxel accuracy is achieved on average.

An objective assessment of the quality of the final images re-assembled in the virtual dissection process is more difficult for patient data in the absence of ground truth as reference. A subjective measure of the registration quality is in the form of ten frame movies with successive frames coming from successive registered images. Perfect registration would result in ten identical frames. The residual movement that is seen is a subjective measure of the registration quality. It can be seen that virtual dissection has significantly reduced motion compared with the unregistered frames.

In the future more patient data will be processed to ensure the procedure is robust in the face of normal variation in patient data.

\section{ACKNOWLEDGMENT}

The authors would like to thank the United Kingdom EPSRC for supporting this work.

\section{REFERENCES}

[1] A. A. Abd Rahni, K. Wells, E. Lewis, M. Guy, and B. Goswami, "An iterative particle filter approach for respiratory motion estimation in nuclear medicine imaging," in Medical Imaging 2011: Image Processing, ser. Proceedings of SPIE, vol. 7962. 
(a) Phase 2
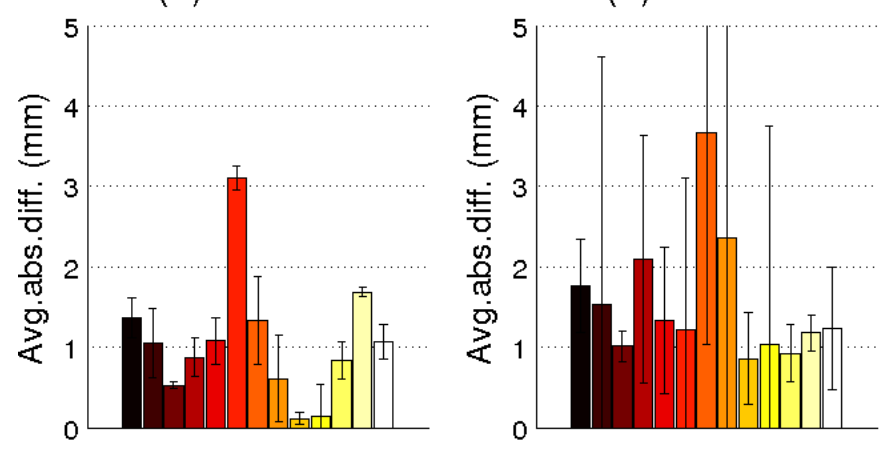

(c) Overall Avg

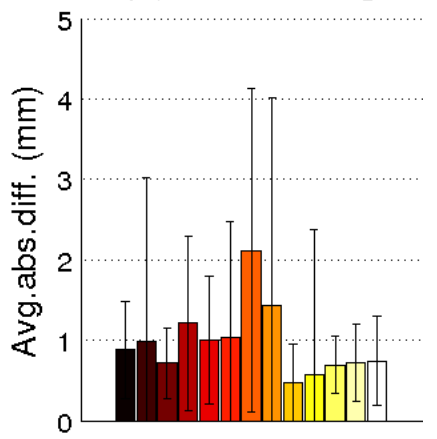

\section{Iterated Closest Points [ICP]}
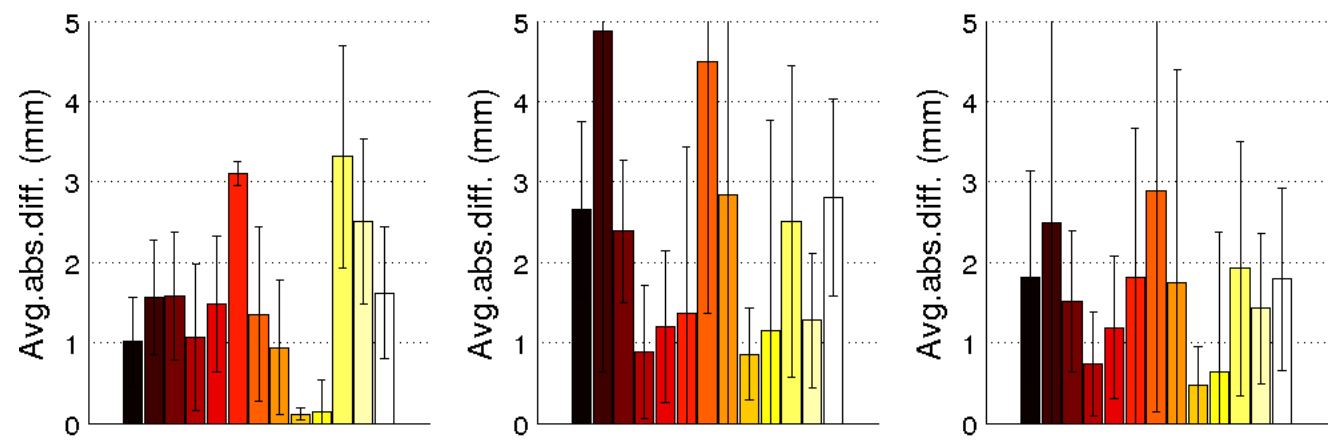

\section{Automated Image Registration [AIR]}

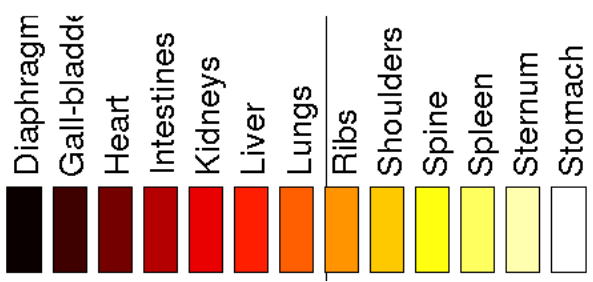

Fig. 4. XCAT data from a single breathing cycle of 10 frames. Individual organs post registration average absolute difference between VDM ground truth and virtual dissection displacement fields(ICP and separately AIR).

[2] P. J. Besl and N. D. McKay, "A method for registration of 3-d shapes," IEEE Transactions on Pattern Analysis and Machine Intelligence, vol. 14, no. 2, pp. 239-256, 1992.

[3] R. P. Woods, S. R. Cherry, and J. C. Mazziotta, "Rapid automated algorithm for aligning and reslicing pet images," Journal of Computer Assisted Tomography, vol. 16, no. 4, pp. 620-633, 1992.

[4] F. Lamare, T. Cresson, J. Savean, C. C. Le Rest, A. J. Reader, and D. Visvikis, "Respiratory motion correction for pet oncology applications using affine transformation of list mode data," Physics in Medicine and Biology, vol. 52, no. 1, pp. 121-140, 2007.

[5] P. Bergstrom and G. Shechter, "Iterated closest points implemenatation," http://www.mathworks.com/matlabcentral/fileexchange/12627iterative-closest-point-method, Checked 18 April 2011.

[6] J. Jones et al, " Registration Quality Movies, " http://www.ee.surrey.ac.uk/Personal/J.Jones, Checked 14 November 2011.

[7] S. Y. Du, N. N. Zheng, G. F. Meng, and Z. J. Yuan, "Affine registration of point sets using icp and ica," IEEE Signal Processing Letters, vol. 15, pp. 689-692, 2008.

[8] W. P. Segars, B. M. Tsui, D. S. Lalush, E. C. Frey, M. A. King, and D. Manocha, "Development and application of the new dynamic nurbsbased cardiac-torso (ncat) phantom," Journal of Nuclear Medicine, vol. 42, no. 5, pp. 7P-7P, 2001, suppl. S. 


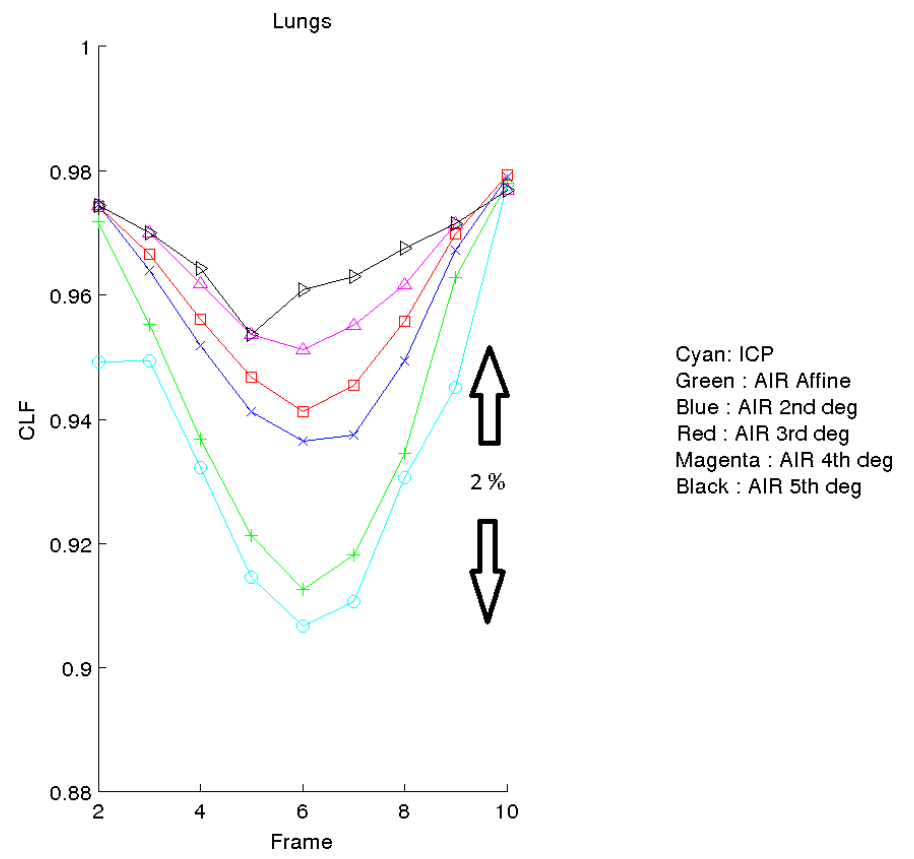

Fig. 5. XCAT data from a single breathing cycle of 10 frames. The effect of varying the degree of the AIR polynomial in the registration of the lungs. For organs which exhibit non-linear deformation, higher order polynomials (more parameters) lead to better CLF (closer to one). 